\title{
Viper bites complicate chronic agrochemical nephropathy in rural Sri Lanka
}

\author{
Anjana Silva ${ }^{1 *}$, Rivikelum Samarasinghe ${ }^{2}$, Senaka Pilapitiya ${ }^{3}$, Niroshana Dahanayake ${ }^{3}$ and Sisira Siribaddana ${ }^{3}$
}

\begin{abstract}
Snakebite is a common occupational health hazard among Sri Lankan agricultural workers, particularly in the North Central Province. Viperine snakes, mainly Russell's viper envenomation, frequently lead to acute renal failure. During the last two decades, an agrochemical nephropathy, a chronic tubulointerstitial disease has rapidly spread over this area leading to high morbidity and mortality. Most of the epidemiological characteristics of these two conditions overlap, increasing the chances of co-occurrence. Herein, we describe four representative cases of viperine snakebites leading to variable clinical presentations, in patients with chronic agrochemical nephropathy, including two patients presented with acute and delayed anuria. These cases suggest the possibility of unusual manifestations of snakebite in patients with Sri Lankan agrochemical nephropathy, of which the clinicians should be aware. It could be postulated that the existing scenario in the Central America could also lead to similar clinical presentations.
\end{abstract}

Keywords: Snakebite, Chronic kidney disease, Agricultural nephropathy, Epidemiology, Anuria

\section{Background}

Sri Lanka has high snakebite related morbidity with 37,000 hospital admissions annually [1]. In the country, snakebite is an occupational hazard associated with agriculture, mostly affecting male farmers [2]. The highest snakebite incidence, mortality and case fatality rates are recorded in the dry zone, (annual rainfall $<2000 \mathrm{~mm}$ ) particularly in the North Central Province (NCP) where farms (Chena), paddy fields and jungles are in close proximity [1,2]. Russell's viper (Daboia russelli) causes $50 \%$ of all snakebites in the dry zone, most of them result in life threatening systemic envenomation characterized by coagulopathy, acute renal failure (ARF) and neurological involvement [1,3,4]. Similarly, Merrem's hump-nosed pit viper (Hypnale hypnale) envenomation may also lead to renal failure [5].

During the last two decades, a chronic kidney disease (CKD) of epidemic proportions disease has been occurring in some areas of NCP and dry zone of Sri Lanka. In a survey of patients that attended the Teaching Hospital Anuradhapura (THA), the main tertiary care center in

\footnotetext{
*Correspondence: nkanjanasilva@gmail.com

1 Department of Parasitology, Faculty of Medicine and Allied Sciences,

Rajarata University of Sri Lanka, Saliyapura 50008, Sri Lanka

Full list of author information is available at the end of the article
}

NCP, $82 \%$ of them did not have an identifiable cause for CKD [6]. The disease had a distinctive epidemiology and affected mainly male paddy farmers that drank water from wells in limited geographical and familial clusters [7]. Pathological findings included progressive tubulointerstitial nephropathy, which indicates environmental or occupational agents [8]. Due to the persistent inability to find a cause for it, this disease was called CKD of unknown etiology. Recently, considering the emerging evidences of agrochemical use as the most possible etiology, it was proposed that the condition should be renamed chronic agrochemical nephropathy (CAN).

At THA, there was a $227 \%$ increase in live discharges and 354\% increase in deaths from 1992 to 2007 among patients with CAN [9]. At present, CAN is the leading cause of death in NCP and its unique distribution suggests an environmental etiology [7,9]. Itai-itai disease due to cadmium poisoning and Balkan nephropathy provoked by exposure to aristolochic acid are two common epidemiologically related kidney diseases due to environmental toxins.

The following factors were associated with CAN: consumption of well water, paddy farming, use of pesticides and past history of snakebite [10,11]. Association of heavy metal poisoning, predominately arsenic, with CAN was 
recently reported [12] and confirmed by the World Health Organization [13]. The above hypothesis relates CAN with contaminated fertilizers and pesticides, which motivated to classify the condition as an agricultural nephropathy.

Unfortunately, CAN shares most of its epidemiological characteristics with snakebite, making rural agricultural communities in NCP of Sri Lanka vulnerable for both these life-threatening conditions. Herein, we report four victims of viperine snakebites aggravated by existing $\mathrm{CAN}$ in male farmers, leading to renal complications.

\section{Case presentations}

\section{Patient 1}

A 72-year-old male farmer diagnosed with CAN grade II was bitten on his right ring finger, while he was handharvesting paddy at 4:30 p.m. on February 20, 2012. After the bite, the patient was admitted to the local hospital. He took with him the dead snake, which was identified as hump-nosed pit viper (Hypnale hypnale) by the attending medical officer. The victim developed pain in the bite site and swelling over the right ring finger. $\mathrm{He}$ had no neurological signs or evidence of spontaneous bleeding. The whole blood clotting time (WBCT) on admission and six hours after had been less than $20 \mathrm{mi}-$ nutes $(<20$ minutes considered to be normal). Indian polyvalent anti-venom (IPA) was not administered.

Since the patient had not passed urine since the snakebite, he was transferred to the Professorial Medical Unit of THA seven hours after the initial admission to the local hospital. On admission to the THA, the patient complained of lower abdominal pain, backache, nausea and several episodes of vomiting. His pulse rate was 80 beats per minute and the blood pressure was 160/90 $\mathrm{mmHg}$. The lungs were clear to auscultation and he had no neurological abnormalities. The patient was catheterized but no urine was seen. His prothrombin time (PT) was $60 \mathrm{~s}$ and international normalization ratio (INR) was 1.5. The blood urea nitrogen $(\mathrm{BUN})$, serum creatinine $(\mathrm{sCr}), \mathrm{Na}^{+}$and $\mathrm{K}^{+}$ were $56 \mathrm{mmol} / \mathrm{L}, 380 \mu \mathrm{mol} / \mathrm{L}, 133 \mathrm{mEq} / \mathrm{L}$, and $4.3 \mathrm{mEq} / \mathrm{L}$ respectively. His electrocardiogram was normal. Despite intravenous (IV) injection of $120 \mathrm{mg}$ of furosemide, the patient remained anuric. His blood pressure, six hours after the admission to THA, was $170 / 90 \mathrm{mmHg}$, pulse rate was $120 \mathrm{~min}^{-1}$ and was having bi-basal crepitations of lungs.

The ultrasound scan of the kidneys showed increased cortical echogenicity and unclear corticomedullary differentiation. Oral furosemide, diltiazem, IV penicillin and cloxacillin were administered. The IV and oral fluid intake was restricted and peritoneal dialysis was initiated on the next day. Following 40 cycles of dialysis, five days after the snakebite, the patient started passing water. Despite further 28 cycles of peritoneal dialysis during the next five days, his BUN and $\mathrm{sCr}$ levels remained elevated above $100 \mathrm{mmol} / \mathrm{L}$ and $800 \mu \mathrm{mol} / \mathrm{L}$. His systolic and diastolic blood pressure values remained above 150 and $90 \mathrm{mmHg}$ respectively. One cycle of hemodyalisis was performed on the $12^{\text {th }}$ day following the bite. His urine output increased to over $500 \mathrm{~mL} /$ day with gradual decline of BUN and sCr levels and the patient was discharged on $15^{\text {th }}$ day following the snakebite.

\section{Patient 2}

A 56-year-old male farmer, a known patient with CAN undergoing treatment, was admitted to THA on September 7, 2012, following a Russell's viper (Daboia russelli) bite on his left foot. The patient was treated with ten vials of IPA for coagulopathy, which was detected by prolonged WBCT, augmented prothrombrin time and INR. The coagulopathy improved with IPA. He had no clinical or biochemical evidence suggestive of ARF and had mild local swelling. He was discharged from the ward on the fourth day, and his level of sCr on that occasion was $124 \mu \mathrm{mol} / \mathrm{L}$.

Three weeks later, on October 2, 2012, the patient was admitted to the local hospital complaining of shortness of breath and anuria for five days. He was immediately transferred to THA and was admitted to the Emergency Treatment Unit. On admission, the patient was conscious, dyspneic, pale and had bilateral leg edema. His pulse was $84 \mathrm{bpm}$ and blood pressure was $180 / 110 \mathrm{mmHg}$. The peripheral oxygen saturation was $91 \%$ and auscultation of chest revealed bibasal crepitations and rhonchi. Although immediately catheterized, no urine was seen. The ECG was normal. Hemoglobin level was $7.8 \mathrm{~g} / \mathrm{dL}$, $\mathrm{pH} 7.37$ and arterial oxygen partial pressure was $89 \mathrm{mmHg}$, while serum $\mathrm{K}^{+}, \mathrm{Na}^{+}$, and blood urea were $6.0 \mathrm{mEq} / \mathrm{L}$, $118 \mathrm{mEq} / \mathrm{L}$ and $24.9 \mathrm{mmol} / \mathrm{L}$, respectively. The patient received respiratory support with $40 \%$ oxygen therapy and was transferred to the Medical Intensive Care Unit where he was treated with IV dextrose insulin and calcium gluconate for hyperkalemia.

On the following day, his blood pressure was 130/ $70 \mathrm{mmHg}$, whilst hemoglobin, $\mathrm{BUN}, \mathrm{sCr}, \mathrm{Na}^{+}$and $\mathrm{K}^{+}$levels were $7.9 \mathrm{~g} / \mathrm{dL}, 31.2 \mathrm{mmol} / \mathrm{L}, 882 \mu \mathrm{mol} / \mathrm{L}, 123 \mathrm{mEq} / \mathrm{L}$ and $6.6 \mathrm{mEq} / \mathrm{L}$, respectively. The chest radiograph showed bilateral pleural effusions. Peritoneal dialysis was initiated and continued for 56 cycles over the next two days. He was transfused with four units of blood. The hyperkalemia and hyponatremia resolved gradually. Following peritoneal dialysis, his BUN, $\mathrm{sCr}, \mathrm{Na}^{+}$and $\mathrm{K}^{+}$levels became $24.6 \mathrm{mmol} / \mathrm{L}, 640 \mu \mathrm{mol} / \mathrm{L}, 126 \mathrm{mEq} / \mathrm{L}$, and $3.9 \mathrm{mEq} / \mathrm{L}$. On the fifth day following admission, patient passed urine. However, on the following day, while aspirating the pleural effusion he developed cardiac arrest and died.

\section{Patient 3}

A 53-year-old male farmer was admitted to a rural hospital, due to a Russell's viper bite on his right foot, at 
7:30 p.m., April 9, 2013. Since the WBCT of the victim was more than 20 minutes, he was immediately transferred to THA. On admission to the Emergency Treatment Unit of THA, at 8:30 p.m., the patient had pain, hemorrhage from the bite site, nausea, swelling, and blurred vision, without any evidence of neurotoxicity. His pulse rate was $60 \mathrm{bpm}$ and the blood pressure was $120 / 70 \mathrm{mmHg}$. The WBCT was normal, but as the laboratory clotting time was 13 minutes, 20 vials of IPA were administered intravenously. His total white cell count was $13600 / \mathrm{uL}$, of which $71 \%$ were granulocytes. The hemoglobin level was $10.8 \mathrm{~g} / \mathrm{dL}$ and the $\mathrm{sCr}$, blood urea, $\mathrm{Na}^{+}$and $\mathrm{K}^{+}$levels were $288 \mu \mathrm{mol} / \mathrm{L}, 11 \mathrm{mmol} / \mathrm{L}$, $141 \mathrm{meq} / \mathrm{L}$ and $3.0 \mathrm{meq} / \mathrm{L}$, respectively. During the next two hours, the patient's urine output was $150 \mathrm{~mL}$. He had no past history of hypertension or diabetes mellitus. Three hours later, the patient developed ptosis, ophthalmoplegia, dysphagia and muscle tenderness. He had no difficulty in breathing. The urine output during the first twelve hours of hospital stay was $1600 \mathrm{~mL}$. At 10:00 a.m. on the next day, his blood pressure was 140/90 $\mathrm{mmHg}$. He had no gross hematuria, normal WBCT and laboratory clotting time was ten minutes. His urinalysis revealed albuminuria and 50 to 60 red blood cells per high power field. The serum $\mathrm{K}^{+}$level was $8.7 \mathrm{mEeq} / \mathrm{L}$ and $\mathrm{Na}^{+}$level was $140 \mathrm{mEq} / \mathrm{L}$.

Following IV calcium gluconate therapy and dextrose insulin, his serum $\mathrm{K}^{+}$level decreased to $3.0 \mathrm{mEq} / \mathrm{L}$. The repeated $\mathrm{sCr}$ and $\mathrm{BUN}$ levels were $325 \mu \mathrm{mol} / \mathrm{L}$ and $15 \mathrm{mmol} / \mathrm{L}$, respectively. His urine output for the next 24 hours was $1650 \mathrm{~mL}$. On the third day of the hospital stay, the $\mathrm{sCr}$ and BUN were $310 \mu \mathrm{mol} / \mathrm{L}$ and $15 \mathrm{mmol} / \mathrm{L}$, respectively. The ultrasound scan showed bilateral contracted kidneys (right kidney pole to pole: $72 \mathrm{~mm}$ and left kidney: $77 \mathrm{~mm}$ ), with blurred corticomedullary differentiation. His blood pressure was $150 / 100 \mathrm{mmHg}$ and the serum electrolyte levels remained within normal range and urine output was $1750 \mathrm{~mL}$ for next 24 hours. The patient was discharged with antihypertensive treatment.

\section{Patient 4}

A 48-year-old male farmer bitten on his right ankle by a Russell's viper on his farm was admitted to the local hospital within ten minutes. Since he had abdominal pain and blurred vision, he was transferred to THA within one hour. On admission at THA, he had mild pain and swelling over his right ankle, headache, nausea and abdominal pain. His WBCT, PT, INR, activated partial thromboplastin time (APTT), white blood cell count (WBC), sCr, BUN on admission were: more than 20 minutes, $19.5 \mathrm{~s}, 1.7,22.75 \mathrm{~s}, 13000 \mu \mathrm{L}^{-1}, 334 \mu \mathrm{mol} / \mathrm{L}$, and $13.6 \mathrm{mmol} / \mathrm{L}$, respectively. His serum $\mathrm{Na}^{+}$and $\mathrm{K}^{+}$ levels were within normal range. The patient developed bilateral ptosis, diplopia, and external ophthalmoplegia rapidly within an hour and was treated with 20 vials of IPA. Six hours later, his clotting profile was normal. However, his sCr and BUN remained elevated $(368 \mu \mathrm{mol} /$ $\mathrm{L}$ and $17.9 \mathrm{mmol} / \mathrm{L}$, respectively) after 24 hours, with normal serum $\mathrm{Na}^{+}$and $\mathrm{K}^{+}$levels. He was normotensive and had normal urinary output and blood glucose levels throughout the hospital stay. The ultrasound scan of both kidneys revealed contracted kidneys with blurred corticomedullary differentiation, indicating chronic kidney disease. The features of neuromuscular paralysis were gradually settled on the fourth day of hospital stay and the patient was discharged from the ward with a clinic follow-up plan for CKD.

\section{Discussion}

The four cases presented herein show that viperine snakebite in a CAN patient may lead to a spectrum of clinical features related to the urinary system, ranging from prolonged anuria to complete normality. The manifestations of the first and second patients included prolonged anuria, lasting more than four days. Although oliguria and anuria are not uncommon presentations of ARF after a snakebite, reports on prolonged anuria are infrequent, apart from a patient bitten by a hump-nosed pit viper who had 16 days of anuria [14]. Interestingly, anuria was present immediately after the bite in the first patient. In the second patient, no biochemical or clinical evidence of an ARF was observed during the initial hospital stay, but manifested three weeks following the bite, indicating a delayed renal injury. In the first patient, prolonged peritoneal dialysis was required to reverse the ARF as the patient had grade two chronic renal failure. In addition, there was mild coagulopathy, compared with severe renal insult.

Although not previously diagnosed, bilateral shrunken kidneys and very high sCr levels on admission, along with the risk factors of being a male farmer suggested that the third patient was suffering from CAN. Interestingly, this patient developed transient hyperkalemia and hypertension and his urine output remained normal without progressing to ARF. However, despite having elevated $\mathrm{sCr}$ and $\mathrm{BUN}$ levels together with coagulopathy and neurotoxicity, the fourth patient did not show any abnormal clinical parameter in the favor of ARF. These different presentations may indicate unpredictability of the renal manifestations in CAN patients after viperine snakebite.

Tubulointerstitial nephritis is considered the major pathological event in CAN. In addition, glomerular sclerosis and vascular changes have also been observed [8]. Viperine venoms are known to cause a varying degree of tubular cell damage throughout the renal tubule and glomerular implications, including glomerulonephritis and vascular changes due to both indirect and direct toxic effects 
[15]. Tubular and glomerular injuries due to viperine venoms were observed in in vitro, in vivo and clinical studies [15-17]. Therefore, it is likely that these venoms cause further damage to already injured glomerular, tubular and vascular structures of kidneys of CAN patients, further compromising their renal function. Low hemoglobin levels observed in first and second patients could be directly related to existing CAN.

As shown by several previous studies, CAN is a male predominate disease $[6,9,10]$. All four patients in this study were male farmers, residing in NCP. Although exact prevalence of CAN is unknown, a recent report of the World Health Organization revealed that $15 \%$ of the 15 to 70 years old population in NCP are affected by CAN [13]. Unfortunately, the vast majority of CAN patients are late presenters at advanced stages of the disease, due to the insidious onset and poor socioeconomic status of the patients [11]. Therefore, it is possible to have a large number of undiagnosed CAN patients in the community. The only available antivenom for snakebite victims in Sri Lanka is Indian polyvalent antivenom (IPA), which is raised against Russell's viper, saw-scaled viper (Echis carinatus), Indian krait (Bungarus caeruleus) and common cobra (Naja naja naja). IPA is not effective in treating hump-nosed pit viper envenomation $[18,19]$. The poor efficacy of IPA against victims of Sri Lankan Russell's viper bite is well-documented [20]. The first patient did not receive IPA and although the second patient did, presumably, neutralization of the Russell's viper venom by IPA may have been ineffective. Therefore, unavailability of safe and effective antivenom to be used against all medically important venomous snakes in Sri Lanka has contributed partly to the complications in both these patients.

During the last two decades, Mesoamerican nephropathy, a chronic kidney disease of uncertain etiology, has rapidly spread over Nicaragua, El Salvador and Costa Rica. Young and middle aged male agricultural workers are the most affected group and many afflicted people are not diagnosed until they reach advanced stage of the disease [21]. Viperine bites are common in this region with $50-80 \%$ of all snakebites being due to terciopelo (Bothrops asper). Most terciopelo victims are young male agricultural workers and acute kidney injury has been reported in $11-17 \%$ of them [22]. Hence, it could be assumed that similar unpredicted clinical presentations and renal outcomes could be expected in snakebite victims in the endemic areas of Mesoamerican nephropathy in Central America.

\section{Conclusion}

Snake envenomation in patients with CAN may precipitate $\mathrm{ARF}$ on already existing renal failure, and may lead to unexpected presentations such as immediate or delayed anuria.

\section{Ethics committee approval}

This study was approved by the Ethics Review Committee of the Rajarata University of Sri Lanka.

\section{Consent}

Written informed consent was obtained from the patients 1, 3 and 4, and from the wife of patient 2 for publication of this case report.

\section{Competing interests}

The authors declare that there are no competing interests.

\section{Authors' contributions}

AS and SS designed the study. AS, RS, ND and SP collected data. SS, SP and ND managed patients. AS drafted the paper. All authors read and approved the final version of the paper.

\section{Acknowledgments}

The authors thank the staff of the THA

\section{Author details}

'Department of Parasitology, Faculty of Medicine and Allied Sciences, Rajarata University of Sri Lanka, Saliyapura 50008, Sri Lanka. ${ }^{2}$ Department of Community Medicine, Faculty of Medicine and Allied Sciences, Rajarata University of Sri Lanka, Saliyapura, Sri Lanka. ${ }^{3}$ Department of Medicine, Faculty of Medicine and Allied Sciences, Rajarata University of Sri Lanka, Saliyapura, Sri Lanka.

Received: 4 May 2014 Accepted: 1 August 2014

Published: 11 August 2014

\section{References}

1. Kasturiratne A, Pathmeswaran A, Fonseka MM, Lalloo DG, Brooker S, de Silva $\mathrm{HJ}$ : Estimates of disease burden due to land-snake bite in Sri Lankan hospitals. Southeast Asian J Trop Med Public Health 2005, 36(3):733-740.

2. De Silva A, Ranasinghe L: Epidemiology of snake-bite in Sri Lanka: a review. Ceylon Med J 1983, 28(3):144-154

3. Jeyarajah R: Russell's viper bite in Sri Lanka. A study of 22 cases. Am J Trop Med Hyg 1984, 33(3):506-510.

4. Kularatne SA: Epidemiology and clinical picture of the Russell's viper (Daboia russelii russelii) bite in Anuradhapura, Sri Lanka: a prospective study of 336 patients. Southeast Asian J Trop Med Public Health 2003, 34(4):855-862.

5. Maduwage K, Isbister GK, Silva A, Bowatta S, Mendis S, Gawarammana I: Epidemiology and clinical effects of hump-nosed pit viper (Genus: Hypnale) envenoming in Sri Lanka. Toxicon 2013, 61:11-15.

6. Athuraliya TN, Abeysekera DT, Amerasinghe PH, Kumarasiri PV, Dissanayake $\checkmark$ : Prevalence of chronic kidney disease in two tertiary care hospitals: high proportion of cases with uncertain aetiology. Ceylon Med J 2009, 54(1):23-25.

7. Wanigasuriya KP, Peiris-John RJ, Wickremasinghe R, Hittarage A: Chronic renal failure in North Central Province of Sri Lanka: an environmentally induced disease. Trans R Soc Trop Med Hyg 2007, 101(10):1013-1017.

8. Nanayakkara S, Komiya T, Ratnatunga N, Senevirathna ST, Harada KH, Hitomi T, Gobe G, Muso E, Abeysekera T, Koizumi A: Tubulointerstitial damage as the major pathological lesion in endemic chronic kidney disease among farmers in North Central Province of Sri Lanka. Environ Health Prev Med 2012, 17(3):213-221.

9. Chandrajith $\mathrm{R}$, Nanayakkara S, Itai K, Aturaliya TN, Dissanayake CB, Abeysekera T, Harada K, Watanabe T, Koizumi A: Chronic kidney diseases of uncertain etiology (CKDu) in Sri Lanka: geographic distribution and environmental implications. Environ Geochem Health 2011, 33(3):267-278.

10. Athuraliya NT, Abeysekera TD, Amerasinghe PH, Kumarasiri R, Bandara P, Karunaratne $\mathrm{U}$, Milton $\mathrm{AH}$, Jones $\mathrm{AL}$ : Uncertain etiologies of proteinuric-chronic kidney disease in rural Sri Lanka. Kidney Int 2011, 80(11):1212-1221.

11. Senevirathna L, Abeysekera T, Nanayakkara S, Chandrajith R, Ratnatunga N, Harada KH, Hitomi T, Komiya T, Muso E, Koizumi A: Risk factors associated with disease progression and mortality in chronic kidney disease of 
uncertain etiology: a cohort study in Medawachchiya, Sri Lanka. Environ Health Prev Med 2012, 17(3):191-198.

12. Jayasumana MACS, Paranagama PA, Amarasinghe MD, Wijewardane KMRC, Dahanayake KS, Fonseka SI, Rajakaruna KDLMP, Mahamithawa AMP,

Samarasinghe UD, Senanayake VK: Possible link of chronic arsenic toxicity with Chronic Kidney Disease of unknown etiology in Sri Lanka. J Nat Sci Res 2013, 3(1):64-73.

13. Mendis S: Chronic Kidney Disease of Unknown Aetiology (CKDu), Sri Lanka, Mission Report. Geneva: World Health Organization; 2012.

14. Varagunam T, Panabokke RG: Bilateral cortical necrosis of the kidneys following snakebite. Postgrad Med J 1970, 46(537):449-451.

15. Sitprija V: Snakebite nephropathy. Nephrology (Carlton) 2006, 11(5):442-448.

16. Chaiyabutr N, Sitprija V: Pathophysiological effects of Russell's viper venom on renal function. J Nat Toxins 1999, 8(3):351-358.

17. Silva A, Gunawardena P, Weilgama D, Maduwage K, Gawarammana I: Comparative in-vivo toxicity of venoms from South Asian hump-nosed pit vipers (Viperidae: Crotalinae: Hypnale). BMC Res Notes 2012, 5:471.

18. Kularatne SA, Ratnatunga N: Severe systemic effects of Merrem's hump-nosed viper bite. Ceylon Med J 1999, 44(4):169-170.

19. Ariaratnam CA, Thuraisingam V, Kularatne SA, Sheriff MH, Theakston RD, de Silva A, Warrell DA: Frequent and potentially fatal envenoming by hump-nosed pit vipers (Hypnale hypnale and H. nepa) in Sri Lanka: lack of effective antivenom. Trans R Soc Trop Med Hyg 2008, 102(11):1120-1126.

20. Phillips RE, Theakston RD, Warrell DA, Galigedara Y, Abeysekera DT, Dissanayaka P, Hutton RA, Aloysius DJ: Paralysis, rhabdomyolysis and haemolysis caused by bites of Russell's viper (Vipera russelli pulchella) in Sri Lanka: failure of Indian (Haffkine) antivenom. Q J Med 1988, 68 (257):691-715.

21. Ramirez-Rubio O, McClean MD, Amador JJ, Brooks DR: An epidemic of chronic kidney disease in Central America: an overview. Postgrad Med J 2013, 89(1049):123-125.

22. Otero-Patiño R: Epidemiological, clinical and therapeutic aspects of Bothrops asper bites. Toxicon 2009, 54(7):998-1011.

doi:10.1186/1678-9199-20-33

Cite this article as: Silva et al:: Viper bites complicate chronic agrochemical nephropathy in rural Sri Lanka. Journal of Venomous Animals and Toxins including Tropical Diseases 2014 20:33.

\section{Submit your next manuscript to BioMed Central and take full advantage of:}

- Convenient online submission

- Thorough peer review

- No space constraints or color figure charges

- Immediate publication on acceptance

- Inclusion in PubMed, CAS, Scopus and Google Scholar

- Research which is freely available for redistribution 